\title{
Effect of free surface deformation on the extractable power of a finite width turbine array
}

\author{
C. R. Vogel ${ }^{a, *}$, G. T. Houlsby ${ }^{\mathrm{a}}$, R. H. J. Willden ${ }^{\mathrm{a}}$ \\ ${ }^{a}$ Department of Engineering Science, University of Oxford, OX1 3PJ, United Kingdom
}

\begin{abstract}
The effect of free surface deformation on the power extracted by a tidal turbine array partially spanning a wide channel is investigated using a theoretical model. It is assumed that two predominant flow scales exist; a turbine-scale flow, and an array-scale flow. The two scales are analysed as quasi-inviscid open channel flow problems, in which conservation of mass, momentum, and energy are considered. Kinematic and dynamic boundary conditions couple the two problems, providing a single multi-scale model. The assumption of constant mass flux becomes exact as the channel width tends to infinity. Array power extraction may be maximised by determining the optimum intra-turbine spacing, which also enhances energy extraction efficiency (ratio of power generated to power removed from the flow). Power extraction and efficiency increase as the Froude number increases, improving array performance in open channels. In the limit of an infinitely wide channel the extracted power depends only on the Froude number and local blockage (ratio of turbine to local flow passage areas). At zero Froude number, the peak power coefficient increases from the LanchesterBetz limit of 0.593 to 0.798 , which occurs when the local blockage ratio is approximately 0.4. For Froude numbers in the range of 0.1 to 0.2 , typical of prospective tidal energy sites, the peak power coefficient increases by an additional $1 \%$ to $4.5 \%$ when the array occupies a negligible fraction of the channel crosssection, increasing further as the array occupies a greater proportion of the channel cross-section.
\end{abstract}

Keywords: Tidal power, Tidal currents, Tidal turbine arrays

1 It is well recognised that the power extracted by a turbine in a channel depends on the blockage ratio, $B$, the ratio of total turbine frontal area to channel cross section. Garrett and Cummins [6] used a one-dimensional model to show that, for a single turbine in a channel with a rigid lid, the maximum power extraction was proportional to $(1-B)^{-2}$. Such a simple relationship was not derived in models incorporating a free-surface, but it was still found that power extraction could be maximised by blocking a large fraction of the channel cross-section [11]. However, this may not be possible for various environmental, technical, and regulatory reasons, and does not account for the reduction of the flow for a given head difference across the channel that occurs as a consequence of the increased resistance imposed on the flow by the turbines. Garrett and Cummins [5] and later Vennell [9] investigated the response of a channel to tidal energy extraction, showing that the flux through the channel reduces when the hydrodynamic drag of the turbines becomes significant relative to the resistance in the undisturbed channel, reducing the energy extracted by the turbines.

It may not be feasible to deploy turbines to span the entire width of the channel due to various economic and regulatory constraints in addition to bathymetric variations and the need to allow passage for shipping and marine life. If the turbine array does not span the entire channel width, then there exist two principal flow dynamics in the channel: the first being flow phenomena that occur close to each tidal turbine; and the second being much larger phenomena that occur on the scale of the array width. The acceleration of the flow in the array bypass due to the aggregate thrust applied by the tidal turbines and corresponding reduction

* Corresponding author

Email address: christopher.vogel@eng.ox.ac.uk (C. R. Vogel) 
in flow speed through the array results in a different estimate of the power that may be extracted by the turbines as compared to analyses in which the turbines span the entire channel width.

Nishino and Willden [7] introduced the concept of scale separation in which array-scale flow phenomena occur more slowly and over longer distances than turbine-scale phenomena, allowing the array-scale and turbinescale flows to be treated as two loosely coupled problems. This allowed the application of the rigid lid model for an array of tidal turbines partially spanning a wide channel. The array-scale problem provided boundary conditions for the turbine-scale model, which was used in turn to determine the thrust applied to the flow, and thus the dynamic coupling to the array scale problem, and the power extraction.

Tidal channels are free surface flows, and as such the water depth must change in order that energy be removed from the flow. The Froude number is typically up to 0.2 in channels of interest for tidal energy development, such as the Pentland Firth or Cook Strait. The rigid lid model assumes that the Froude number is negligible, restricting its application to low Froude number channels and to conditions where the impact of the turbine array on the flow is small, which may not accurately reflect sites of current interest. The reduction in water depth and acceleration of the downstream flow in the turbine (or array) wake will be particularly important when considering multiple rows of turbines. Linear Momentum Actuator Disc Theory (LMADT) has been applied to turbines in constrained flow with a deformable free surface [11] and extended to consider downstream mixing [3] to allow analytic modelling of turbine arrays where free surface deformation may be important and the turbine array spans the full channel width.

In the present work, a general model incorporating a free surface into the scale separation framework is developed to provide an analytic model of a turbine array partially spanning a wide channel. Particular consideration is given to the limiting case of an infinitely wide channel (array blockage tends to zero), as many tidal arrays may only occupy a small fraction of a very much wider tidal channel. The analytic model is derived with respect to an upstream reference velocity, and may be embedded within a larger model, such as that of [5], to account for changes in the mass flow rate due to increased resistance of the flow by the turbines. Note however that the assumption of constant mass flux and upstream Froude number becomes exact in the case of turbines operating in an infinitely wide channel, which may be a suitable approximation for many tidal arrays, removing the necessity for a larger channel model. Furthermore, it is assumed that the flow is inviscid and that no mixing occurs other than in the mixing zones of the two models described below.

\section{Model}

A tidal turbine array of width $L$, composed of $n$ turbines, each of frontal area $A_{L}$, partially spanning a channel of width $W_{C}$ and depth $h_{0}$ is shown in figure 1. There is a flow passage of width $b$ encompassing each turbine, such that $L=n b$. Energy is conserved everywhere except for the discontinuity in static head across the turbines and in the mixing zones at the turbine and array scales.

At the turbine-scale (figure 1(a)), the inviscid, incompressible flow at station 1 slows as it approaches the upstream side of the actuator disc at station 2. A step-change in static head occurs in the streamtube encompassing the turbine between stations 2 and 3 as a result of the applied thrust. Downstream of station 3 , the flow in the wake of the disc continues to slow until hydrostatic pressure variation is achieved at station 4. The expansion of the streamtube encompassing the actuator disc between stations 1 and 4 results in an acceleration of the flow outside the streamtube, which results in a velocity differential between core and bypass flows at station 4 . These velocities necessarily mix between stations 4 and 5 to produce a uniform flow speed at 5 , where the pressure variation is again hydrostatic. The turbine-scale model results in an increase in velocity from 1 to 5 and a decrease in static head.

It is assumed that the velocity $u_{0}$ at the upstream boundary of the channel is spatially uniform and unaltered by turbine operation. The water depth is permitted to vary along the length of the channel. At the array scale (figure 1(b)), the inviscid, incompressible flow upstream of the turbine array slows, resulting in an increase in static head between stations 0 and 1. A static head and velocity discontinuity is established between stations 1 and 5 , as described above, and the streamtube encompassing the array expands as the flow speed reduces 


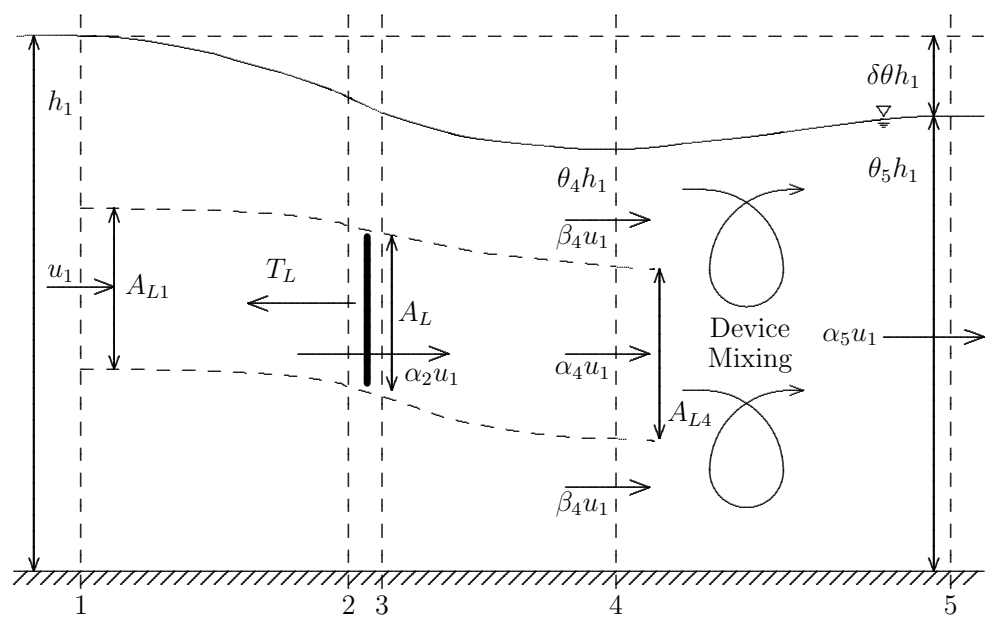

(a) Turbine-scale flow mixing and free surface change.

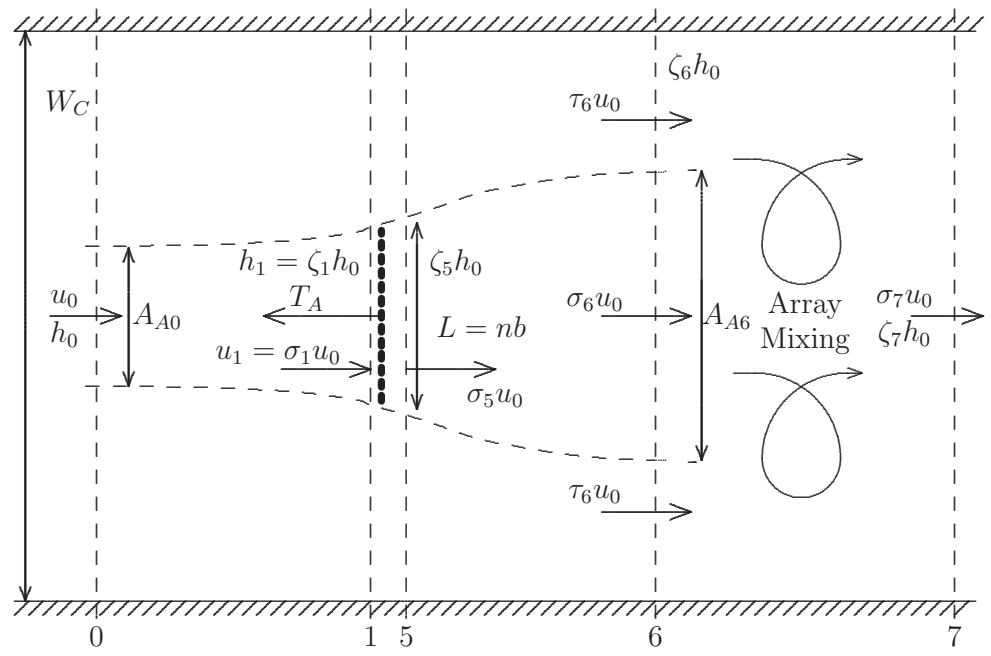

(b) Array-scale flow mixing.

Figure 1: The scale-separated partial array model: (a) turbine-scale, and (b) plan view of the array-scale.

until a spanwise uniform hydrostatic pressure variation is achieved across the width of the channel at station 6. The expansion of the streamtube causes acceleration of the flow around the array, resulting in a velocity differential at station 6 between array core and bypass flows, which necessarily remix to yield a spanwise uniform flow speed and hydrostatic pressure variation at the outlet at station 7 .

Flow phenomena occur over two principal length scales; the turbine diameter $D$, and the array length $L$. Turbine-scale energy extraction and mixing scales on the turbine diameter, whereas the flow around the array scales on the array width. If there are many turbines in the array, then $L \gg D$ and the three-dimensional turbine-scale flow occurs over a much shorter spatial and temporal scale than flow around the array, which will tend to being two-dimensional, as the array width is much greater than the channel depth. The difference in the turbine and the array length scales is assumed to be sufficiently large such that turbine-scale core and bypass flow wake mixing is completed upstream of cross-stream depth equalisation (hydrostatic pressure recovery) in the array scale problem. The partial fence problem may thus be considered in terms of the two loosely coupled quasi-inviscid problems shown in figure 1; a turbine-scale problem and an array-scale problem. Each problem is evaluated separately as an open channel flow, adapting the open channel model of [11] to the two-scale partial fence problem. 
The turbine-scale and array-scale problems are non-dimensionalised by scaling the velocities and static heads in terms of the upstream height and velocity at station 0 in the array-scale problem and station 1 in the turbine-scale problem. In the turbine-scale problem, non-dimensional heights are denoted with $\theta_{i}=h_{i} / h_{1}$, and non-dimensional speeds with $\alpha_{i}=u_{i} / u_{1}$ in the core and $\beta_{i}=u_{i} / u_{1}$ in the bypass flow regions. In the array-scale problem, $\zeta_{i}=h_{i} / h_{0}$ denotes non-dimensional heights, and $\sigma_{i}=u_{i} / u_{0}$ and $\tau_{i}=u_{i} / u_{0}$ the core and bypass non-dimensional flow speeds respectively.

The turbine-scale problem considers a flow passage of centre-to-centre turbine spacing $b$. The water depth and uniform velocity at station one are the same in the array- and turbine-scale problems. The flow speed reduces to $\alpha_{2} u_{1}$ as it passes through the turbine, reducing further to $\alpha_{4} u_{1}$ in the wake of the turbine. Energy is conserved through the core flow passage except across the turbine disc. Meanwhile, the energy conserving flow bypassing the turbine continuously accelerates to reach $\beta_{4} u_{1}$ at station four, where hydrostatic pressure variation is recovered across core and bypass flows resulting in a static head $\theta_{4} h_{1}$. Downstream of four, the turbine core and bypass flows remix, resulting in energy loss, leading to a new uniform velocity $\alpha_{5} u_{1}$ and water depth $\theta_{5} h_{1}$ at station five. Energy extraction from the sub-critical flow results in the free surface at the outlet of the turbine-scale model being lower than that at the inlet, with a corresponding increase in velocity to conserve mass flux. Thus the power available to the turbine is a function of both the upstream flow speed and the far field change in free surface elevation. The turbine-scale downstream flow is imposed on the array-scale model at station five in figure $1(\mathrm{~b})$.

The device occupies some fraction of the local flow passage, leading to the definition of the local turbine blockage, $B_{L}$ as:

$$
B_{L}=\frac{\text { Turbine frontal area }}{\text { Flow passage cross-sectional area }}=\frac{A_{L}}{b h_{1}} .
$$

The thrust coefficient $C_{T L}$ of the turbine is defined as the turbine thrust $T_{L}$ normalised on the upstream dynamic pressure in the turbine scale problem of the flow projected onto the turbine frontal area:

$$
C_{T L}=\frac{T_{L}}{\frac{1}{2} \rho u_{1}^{2} A_{L}} .
$$

The power coefficient $C_{P L}$ of the turbine is the product of the turbine thrust and the flow speed through the turbine, and is normalised on the kinetic flux of the flow upstream of the turbine in the turbine scale problem projected onto the turbine frontal area:

$$
C_{P L}=\frac{\alpha_{2} u_{1} T_{L}}{\frac{1}{2} \rho u_{1}^{3} A_{L}}=\alpha_{2} C_{T L} .
$$

The flow in the array-scale problem is assumed to be a two-dimensional shallow water flow, as the cross-stream extent of the channel is much greater than the depth of the channel. The array, composed of $n$ turbines, is a fence of width $L=n b$ which extends the entire depth of the flow and has a frontal area $A_{A}=L h_{1}$. n must be sufficiently large to satisfy the scale separation assumption. Furthermore, the flow across the face of the fence is assumed to be uniform, thus we neglect any array end effects. The requirement that this places on $n$ has been investigated through comparison to 3D computed fence flows with the result that differences in analytic model prediction fall below $6 \%$ for $n \geq 8$ for moderate blockage ratios [8]. The present model will tend to this limit as the Froude number reduces to 0, and it is expected that agreement will improve further as Froude number increases as the effect of gravity is to help restore the hydrostatic pressure gradient at station four. This reduces the streamwise length of the device-scale problem, increasing the scale separation between the device and array scale problems, improving the agreement of the analytic model with numerical experiments, such as [8].

The thrust imposed by the array of turbines causes the inviscid flow to decelerate to $\sigma_{1} u_{0}$ in front of the array, providing the upstream boundary to the turbine-scale model. There is a change in height and velocity due to the energy extraction of the turbines, resulting in further acceleration and height reduction to station five at exit from the turbine-scale problem, which provides the internal upstream boundary condition to the array mixing problem. The array-scale wake then expands further downstream until there is lateral hydrostatic 
pressure equalisation between the array core and bypass flows at station six. The two array flows remix and produce a uniform velocity $\sigma_{7} u_{0}$, greater than the inlet velocity due to mass flux conservation in response to the decrease in water depth to $\zeta_{7} h_{0}$ resulting from energy removal from the sub-critical flow.

The array blockage ratio, $B_{A}$, is the fraction of the channel blocked by the array:

$$
B_{A}=\frac{\text { Array frontal area }}{\text { Channel cross-sectional area }}=\frac{A_{A}}{W_{C} h_{0}}
$$

The thrust imposed by the array is the sum of the thrust of the turbines in the array, leading to the array thrust coefficient $C_{T A}$ :

$$
C_{T A}=\frac{T_{A}}{\frac{1}{2} \rho u_{0}^{2} A_{A}}=\frac{n T_{L}}{\frac{1}{2} \rho u_{0}^{2} n b h_{1}}=\sigma_{1}^{2} B_{L} C_{T L} .
$$

$$
\text { Tor }
$$

The array power coefficient $C_{P A}$ is the product of the array thrust and the flow speed through the array, normalised on the kinetic flux of the upstream flow projected onto the array:

$$
C_{P A}=\frac{T_{A} u_{1}}{\frac{1}{2} \rho u_{0}^{3} A_{A}}=\frac{n T_{L} u_{1}}{\frac{1}{2} \rho u_{0}^{3} n b h_{1}}=\sigma_{1}^{3} B_{L} C_{T L} .
$$

Finally, it should be noted that the global blockage ratio, $B_{G}$, is the ratio of the total turbine area to the cross section of the channel:

$$
B_{G}=\frac{\text { Total turbine frontal area }}{\text { Channel cross-sectional area }}=\frac{n A_{L}}{W_{C} h_{0}}=B_{A} B_{L} .
$$

\subsection{Turbine-scale model}

An analytic model for an actuator disc in an open channel flow was developed to extend the analysis of Garrett and Cummins [6] to cases where free surface deformation may be important in the vicinity of the turbine [11]. Whelan et al.'s model was subsequently extended by Draper et al. to include the viscous mixing that occurs between stations four and five in figure 1(a) as a result of the shear between the core and bypass flows [3]. Mixing in this region is important because it provides the downstream turbine-scale steady state description that completes the coupling of the turbine-scale and array-scale models.

Whelan et al.'s open channel model and Draper et al.'s extension to include the downstream mixing define, respectively, non-dimensional 'near field' and 'far field' turbine-scale problems. The near field describes the flow between stations one and four and the far field describes the flow between stations one and five in terms of the turbine thrust coefficient, blockage, and Froude number, $F r_{L}=u_{1} / \sqrt{g h_{1}}$.

Following [11] to solve the near field problem, applying conservation of mass between stations one and four in the bypass streamtube yields:

$$
u_{1}\left(h_{1} b-A_{L 1}\right)=u_{1} \beta_{4}\left(h_{1} b \theta_{4}-A_{L 4}\right),
$$

where $A_{L 1}$ and $A_{L 4}$ are the cross-sectional areas of the core flow stream tube at stations one and four respectively. In the core flow streamtube, continuity requires:

$$
u_{1} A_{L 1}=\alpha_{2} u_{1} A_{L}=\alpha_{4} u_{1} A_{L 4}
$$

Equations (8) and (9) are combined to give the turbine induction factor $\alpha_{2}$ :

$$
\alpha_{2}=\frac{\alpha_{4}\left(\beta_{4} \theta_{4}-1\right)}{B_{L}\left(\beta_{4}-\alpha_{4}\right)} .
$$

Bernoulli's equation in the bypass is used to determine the non-dimensional static head $\theta_{4}$ where the hydrostatic pressure equalises in the bypass and core streamtubes at station four:

$$
\theta_{4}=1-\frac{1}{2} \operatorname{Fr}_{L}^{2}\left(\beta_{4}^{2}-1\right)
$$


Here, Bernoulli's equation is applied to the core and bypass flows upstream and downstream of the actuator disc to yield the pressure drop across the disc, which integrated over the surface of the disc, yields the thrust exerted by the disc on the flow:

$$
T_{L}=\frac{1}{2} \rho u_{1}^{2} A_{L}\left(\beta_{4}^{2}-\alpha_{4}^{2}\right) .
$$

The power extracted locally by the turbine, equation (3), may be determined by combining equations (10) and (12). Conservation of momentum of the turbine passage flow between stations one and four may also be used to determine the thrust of the actuator disc:

$$
\begin{aligned}
T_{L}=\int_{0}^{h_{1}} \rho g b z d z & -\int_{0}^{\theta_{4} h_{1}} \rho g b z d z \\
& +\rho u_{1}^{2} b h_{1}\left(1-B_{L} \alpha_{2}\right)\left(1-\beta_{4}\right)+\rho u_{1}^{2} b h_{1} B_{L} \alpha_{2}\left(1-\alpha_{4}\right),
\end{aligned}
$$

which yields a quartic in $\beta_{4}$ when equated with equation (12):

$$
\begin{aligned}
F r_{L}^{2} \beta_{4}^{4}+4 \alpha_{4} F r_{L}^{2} \beta_{4}^{3}+2\left(2 B_{L}-2-F r_{L}^{2}\right) \beta_{4}^{2} & +4\left(2-2 \alpha_{4}-F r_{L}^{2} \alpha_{4}\right) \beta_{4} \\
& +\left(8 \alpha_{4}-4+F r_{L}^{2}-4 \alpha_{4}^{2} B_{L}\right)=0 .
\end{aligned}
$$

Additionally, the far field turbine scale solution is found by considering the far field change in momentum between stations one and five. This yields a cubic in $\delta \theta=\theta_{1}-\theta_{5}$, where it should be noted that $\theta_{1} \equiv 1$ and that continuity requires $\alpha_{5}=1 /(1-\delta \theta)$ :

$$
\delta \theta^{3}-3 \delta \theta^{2}+\left(F r_{L}^{2}\left(B_{L} C_{T L}-2\right)+2\right) \delta \theta-F r_{L}^{2} B_{L} C_{T L}=0 .
$$

The solution procedure for the near field depends on the parameter chosen to specify the turbine's operating conditions. Whelan et al. specify the wake factor $\alpha_{4}$, yielding the quartic for $\beta_{4}$ in equation (14) [11], which is mathematically more convenient than the alternative of specifying the thrust coefficient $C_{T L}$ which leads to a more complicated solution involving radicals in $C_{T L}$. However, it may be more physically meaningful to specify the thrust coefficient as the independent variable in the system of equations. Furthermore, choosing $C_{T L}$ as the turbine parameter allows the direct decoupling of the near field and far field turbine-scale problems, in addition to the quasi-independent turbine-scale and array-scale problems.

The far field problem, derived from [3], provides the uniform flow speed and depth at the downstream boundary of the turbine-scale problem that is then used as the inner upstream boundary condition to the array-scale mixing problem. The head difference between stations one and five, $h_{1}-h_{5}=\delta \theta h_{1}=\delta \zeta h_{0}$, is made to be consistent between the array- and turbine-scale problems, and the coupling between the two problems can be written by expressing the far-field turbine-scale problem in equation (15) in terms of arrayscale variables at station 0 , noting that the array Froude number, $F r_{A}=u_{0} / \sqrt{g h_{0}}$, as:

$$
\delta \zeta^{3}-3 \zeta_{1} \delta \zeta^{2}+\left(\sigma_{1}^{2} F r_{A}^{2}\left(B_{L} C_{T L}-2\right)+2 \zeta_{1}\right) \zeta_{1} \delta \zeta-\zeta_{1}^{2} \sigma_{1}^{2} F r_{A}^{2} B_{L} C_{T L}=0
$$

\subsection{Array-scale model}

The array-scale model is comprised of array-scale core and bypass flows as well as an array-scale mixing zone. Turbine-scale model energy extraction results in a static head difference across the turbine plane, whilst in the array-scale problem stations one and five, encapsulating the turbine-scale problem, are collocated, enabling complementary velocity and height discontinuities as well as a static head difference. The additional equations required to close the array-scale problem are found by considering equation (16) which provides the far-field change in water depth and flow speed in the turbine-scale model, coupling the two models.

Near field and far field solutions exist to the array-scale model, and are of similar form to those for the turbinescale model. The primary difference is the near field array-scale solution requires the far field solution of the turbine-scale model to close the system of equations describing the array-scale model due to the velocity discontinuity across the array in addition to the head discontinuity. The effect of the turbine array in the 
array-scale problem may be specified in terms of one of a number of control parameters, such as $C_{T A}, \sigma_{6}$, or $\delta \zeta_{A}=1-\zeta_{7}$. As the array thrust is linked to the turbine thrust through equation (5), it is convenient to specify $C_{T A}$ to solve the array-scale problem and then match the array thrust coefficient with the appropriate turbine thrust coefficient to determine the power extracted by the array.

Solving the near field problem, conservation of mass in the array core flow between stations zero and six requires:

$$
u_{0} h_{0} A_{A 0}=\sigma_{1} \zeta_{1} u_{0} h_{0} L=\sigma_{5} \zeta_{5} u_{0} h_{0} L=\sigma_{6} \zeta_{6} u_{0} h_{0} A_{A 6} .
$$

An expression for $\zeta_{6}$ is found from conservation of mass in the array bypass flow passage between stations zero and six, giving:

$$
\zeta_{6}=\frac{B_{A} \sigma_{1}}{\sigma_{6}}+\frac{1-B_{A} \sigma_{1}}{\tau_{6}},
$$

from which an expression for $\sigma_{1}$ that is similar to equation (10) for $\alpha_{2}$ may be derived:

$$
\sigma_{1}=\frac{\sigma_{6}\left(\zeta_{6} \tau_{6}-1\right)}{B_{A}\left(\tau_{6}-\sigma_{6}\right)}
$$

The Bernoulli equation for the array core and bypass flow are respectively:

$$
\zeta_{1}=1-\frac{1}{2} F r_{A}^{2}\left(\sigma_{1}^{2}-1\right), \quad \zeta_{5}=\zeta_{6}-\frac{1}{2} F r_{A}^{2}\left(\sigma_{6}^{2}-\sigma_{5}^{2}\right)
$$

$$
\zeta_{6}=1-\frac{1}{2} \operatorname{Fr}_{A}^{2}\left(\tau_{6}^{2}-1\right),
$$

analogous to equation (11). Integrating the pressure drop across the surface of the array yields the array thrust:

$$
T_{A}=\frac{1}{2} \rho g L h_{0}^{2}\left(\zeta_{1}^{2}-\zeta_{5}^{2}\right)
$$

The difference in the form of this equation to that of equation (12) for the turbine thrust arises from the change in water depth that occurs across the array. Bernoulli's equation, (20) and (21) is again used to eliminate the terms due to pressure (here, $\zeta_{1}$ and $\zeta_{5}$ ), but the quadratic form of the equation leads to a more complicated expression in terms of the induction factors $\sigma_{1}, \sigma_{5}, \sigma_{6}$, and $\tau_{6}$, than occurs in the turbine-scale problem.

Near field conservation of momentum from stations zero to six yields:

$$
1-\zeta_{6}^{2}-F_{A}^{2} B_{A} C_{T A}=2 F_{A}^{2}\left(B_{A} \sigma_{1}\left(\sigma_{6}-\tau_{6}\right)+\left(\tau_{6}-1\right)\right) .
$$

The system of equations defining the near field array-scale problem, equations (17) to (23), closed by equation (16) from the turbine-scale model, cannot be solved analytically due to the static head and velocity discontinuity between stations one and five. The equations must instead be solved numerically. As the equations have a shallow gradient close to the physically admissible roots, a bounded root-finding algorithm is required to solve the system. The Brent-Dekker method, which combines the bisection, secant and inverse quadratic interpolation methods [1], was employed to provide guaranteed convergence to the roots of the system of equations. Selection of the appropriate root is guided by the requirement for a physically meaningful solution to the problem: $0 \leq \sigma_{6} \leq \sigma_{1} \leq 1$, and $\tau_{6} \geq 1$, which infers positive power extraction and no flow reversal.

As with the far field turbine-scale problem, the far field array-scale problem is again solved by considering conservation of momentum in the far field between stations zero and seven, noting from continuity that $\sigma_{7}=1 /\left(1-\delta \zeta_{A}\right)$, yielding:

$$
\delta \zeta_{A}^{3}-3 \delta \zeta_{A}^{2}+\left(F r_{A}^{2}\left(B_{A} C_{T A}-2\right)+2\right) \delta \zeta_{A}-F r_{A}^{2} B_{A} C_{T A}=0,
$$

where $\delta \zeta_{A}$ is the overall depth change in the channel. The far field effects of energy extraction can be determined from equation (24) without any further analysis of the problem. However, in order to determine the power extracted by the array of turbines, it is necessary to solve both scales of the problem in order to determine $\alpha_{2}$ and thus the power extracted at the turbine-scale. 

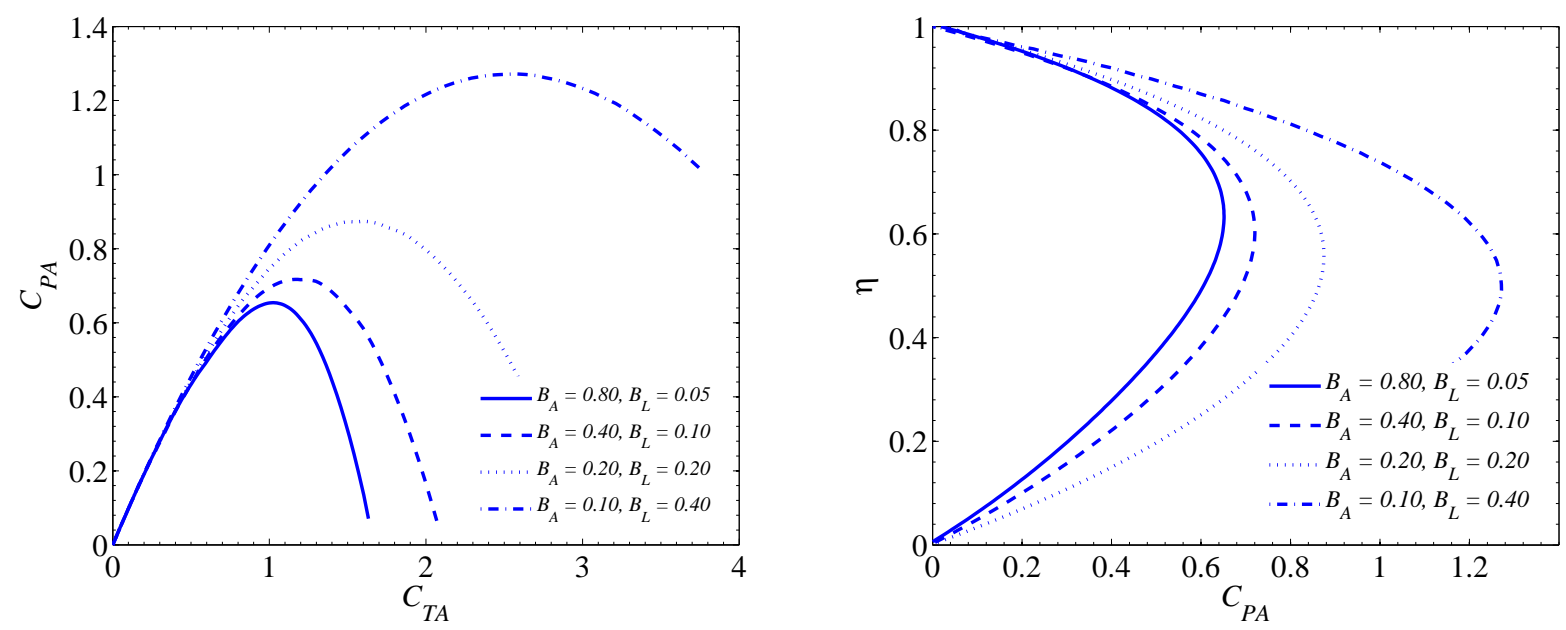

Figure 2: Effect of array and local blockage ratios, $B_{A}$ and $B_{L}$, on thrust $C_{T A}$ and power $C_{P A}$ coefficients (left) and basin efficiency $\eta$ (right) for global blockage $B_{G}=0.04$ and Froude number $F r_{A}=0.10$.

\section{General results}

The effect of varying turbine spacing is shown in figure 2, where the array blockage may be interpreted as the fraction of the channel occupied by the turbine array, the local blockage as the proportion of the array occupied by the turbines, and the global blockage is the proportion of the entire channel area occupied by turbines, which is kept constant.

As the local blockage increases and the array blockage decreases the thrust on the turbines and the power extracted increases. When the turbines are placed very far apart, as in the $B_{A}=0.80, B_{L}=0.05$ case, the peak $C_{P A}$ is relatively low, albeit greater than Betz, due to the array thrust, $C_{T A}$, being relatively low. This is because the flow is relatively free to divert around each turbine, and therefore the thrust, a function of local through turbine plane flow speed squared, that can be applied to the flow is limited as the velocity through each turbine drops quickly. A greater power coefficient is achieved by increasing the local blockage ratio (which necessitates a reduction in the array blockage to maintain the global blockage ratio $B_{G}=0.04$ ), resulting in the peak array power coefficient approximately doubling from $C_{P A, \max }=0.65$ for $B_{A}=0.80, B_{L}=0.05$ (e.g., $9.5 \mathrm{D}$ lateral inter-turbine spacing in a water depth of $1.5 \mathrm{D}$ ) to $C_{P A, \max }=1.27$ when $B_{A}=0.10, B_{L}=0.40$ (e.g., $0.3 \mathrm{D}$ lateral inter-turbine spacing in a water depth of $1.5 \mathrm{D}$ ). As the local blockage increases, there is greater resistance to flow diverting around each turbine due to proximity of neighbouring turbines, so that the velocity through the turbines is higher, thus the thrust and correspondingly the power remain higher too.

It is informative to introduce an additional measure of efficiency, often called the 'basin efficiency', which has been proposed to supplement the traditional efficiency measure described by the power coefficient, and measures the efficiency of power extraction to the total power removed from the flow, rather than the power available to the turbines. The basin efficiency is the ratio of the power extracted by the turbines to the total power removed from the flow, which is comprised of the power extracted by the turbines and the power lost in the viscous mixing regions. The total power removed from the flow can be found from the total change in head, $\dot{m} g\left(H_{\text {inlet }}-H_{\text {outlet }}\right)$, at each scale. Following equation 12 in [3], this yields an expression for the basin efficiency:

$$
\begin{aligned}
\eta & =\frac{\text { Power extracted by turbines }}{\text { Power extracted by turbines and dissipated in mixing }} \\
& =\frac{\sigma_{1} \alpha_{2}\left(1-\delta \zeta_{A}\right)(1-\delta \theta)\left((2-\delta \theta)(1-\delta \theta)-2 F r_{L}^{2}\right)\left(\left(2-\delta \zeta_{A}\right)\left(1-\delta \zeta_{A}\right)-2 F r_{A}^{2}\right)}{\left(2\left(1-\delta \zeta_{A}\right)^{2}+F r_{A}^{2}\left(\delta \zeta_{A}-2\right)\right)\left(2(1-\delta \theta)^{2}+F r_{L}^{2}(\delta \theta-2)\right)},
\end{aligned}
$$




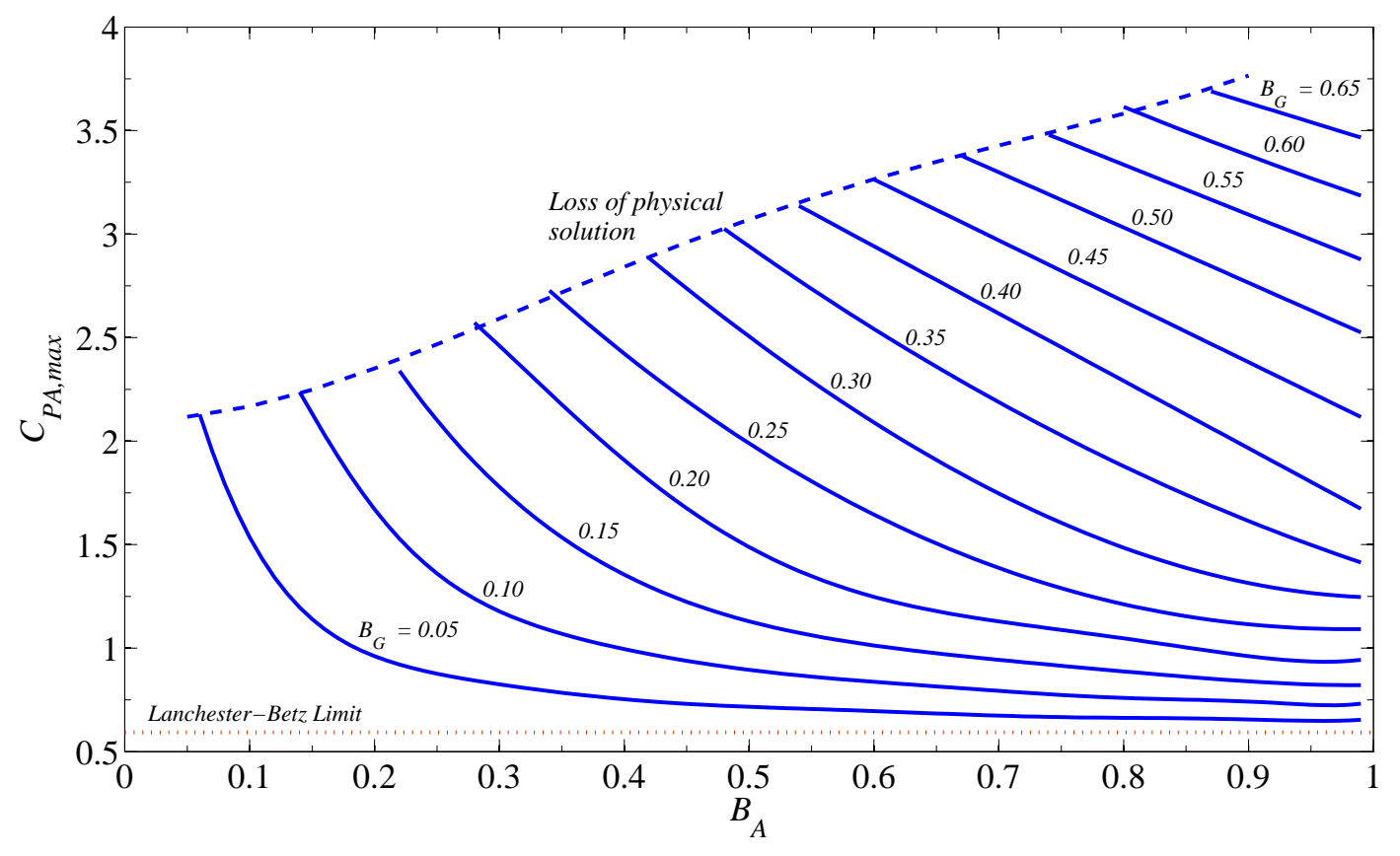

Figure 3: Effect of increasing the array blockage $B_{A}$ on the maximum power coefficient $C_{P, \max }$ for a range of constant global blockage ratios $B_{G}$ at Froude number $F r_{A}=0.10$. The loss of physical solution corresponds to complex roots for the bypass induction factor $\beta_{4}$.

Increasing $B_{L}$ causes the basin efficiency to decrease at peak power coefficient, as the larger thrusts imposed at higher $B_{L}$ cause greater shear between the turbine wake and bypass flow, which in turn increases the energy dissipation in the mixing region. The basin efficiency at the peak power coefficient $C_{P A}=0.65$ in the $B_{A}=0.80, B_{L}=0.05$ case is $\eta=0.64$, so that $1 \mathrm{MW}$ extracted by the turbine removes about $1.6 \mathrm{MW}$ from the flow. This contrasts with the case where $B_{A}=0.10, B_{L}=0.40$ for which $\eta=0.50$ when $C_{P A}=1.27$, so that $1 \mathrm{MW}$ extracted by the turbine removes $2 \mathrm{MW}$ from the flow. However, higher local blockage ratios are more efficient for a given array power coefficient, such that the basin efficiency for closely spaced turbines, $B_{A}=0.10, B_{L}=0.40$, is $\eta=0.85$ at $C_{P A}=0.65$ compared to $\eta=0.64$ when widely spaced $B_{A}=0.80, B_{L}=0.05$. For a given level of power delivery, the velocity through the turbine is higher when the turbine blockage is higher due to the reduced deflection of the flow, and therefore there are lower shear stresses between the bypass flow and turbine wake, resulting in less energy dissipation in the mixing region, improving the basin efficiency. Equivalently, to achieve the same power, less thrust is required at closer turbine spacings.

The general effect of the global blockage on the maximum power coefficient is shown in figure 3 . In all cases, even the case of turbines uniformly distributed across the channel width, i.e. $B_{A}=1$, it is possible to improve upon the Lanchester-Betz limit due to the blockage of the channel. For a given global blockage, i.e. fixed number of turbines, as $B_{A}$ is reduced and the fence only partially occupies the channel width, local blockage $B_{L}$ is increased and there is greater resistance to deflection of the flow into the turbine bypasses. Thus the turbines can support a greater pressure drop across them, increasing the power and thrust at the peak operating point as local blockage increases. This maximum power occurs at reduced velocity through the turbine plane as $B_{L}$ increases and the bypass becomes more constrained, albeit with a higher flow velocity through the bypass. This leads to the decrease in $\eta$ at maximum power with increasing blockage noted in the previous figure. However, if a specific $C_{P A}$ is targeted, efficiency $\eta$ is seen to increase with $B_{L}$, and for a fixed $\eta$, maximum $C_{P A}$ is delivered at maximum $B_{L}$. Importantly, figure 3 shows that the improvement in array performance as $B_{A}$ is reduced is more significant when $B_{G}$ is small, which is of practical significance in the design of realistic turbine arrays. For example, this indicates that for a fixed number of turbines in a large 

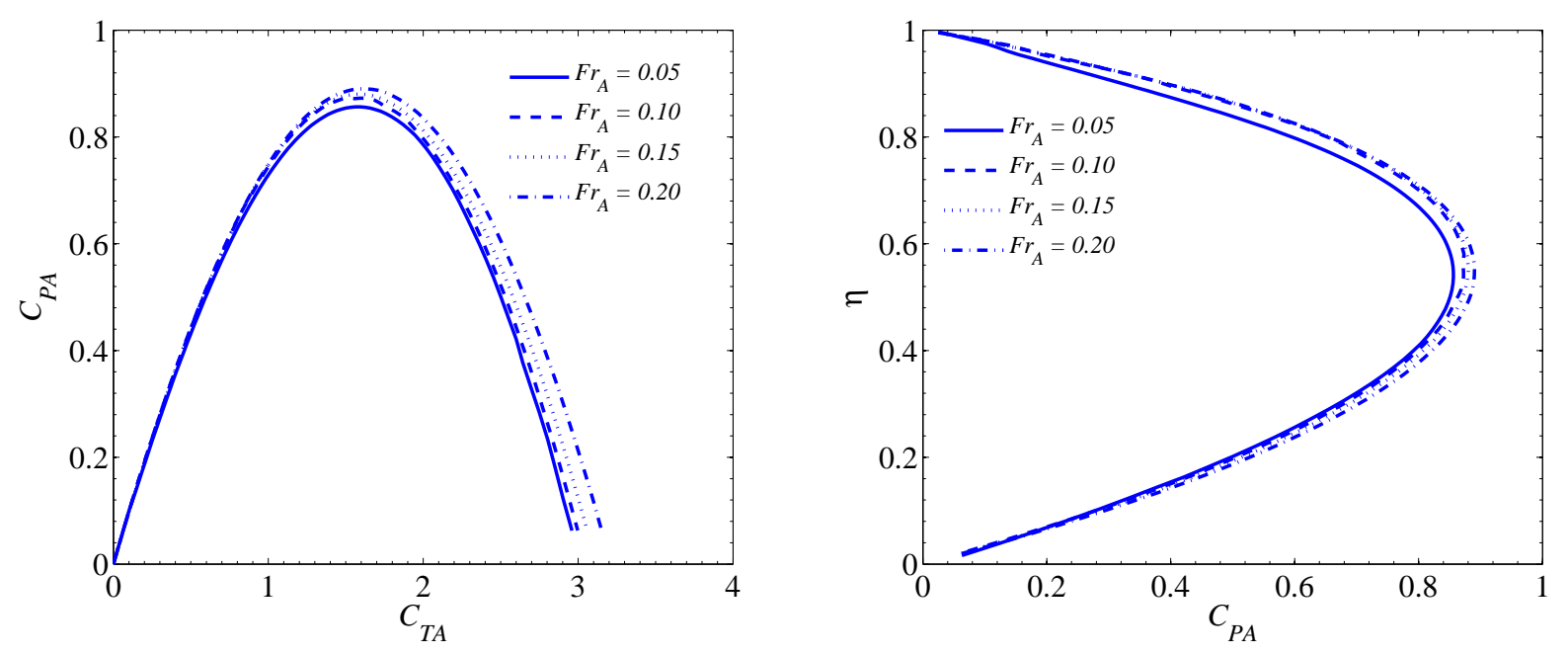

Figure 4: Effect of increasing the Froude number $F r_{A}$ on the thrust $C_{T A}$ and power coefficients $C_{P A}$ (left) and basin efficiency $\eta$ (right) for array blockage $B_{A}=0.20$ and local blockage $B_{L}=0.20$.

channel that achieves a low global blockage, reducing the spacing between turbines and thus the proportion of the channel width occupied by turbines, i.e. $B_{A}$, leads to improved performance. The limiting case of $B_{A} \rightarrow 0$ is discussed further in the next section.

The maximum blockage ratio than can be achieved is limited by how closely turbines can be packed and by clearance requirements from the seabed, other turbines and the surface. A further constraint is imposed in the turbine-scale model to prevent the loss of a physically meaningful solution to the model, where it is required that $0 \leq \alpha_{2} \leq \alpha_{4} \leq 1, \beta_{4} \geq 1$, and $C_{T L} \geq 0$. The breakdown of physical solutions occurs first in the turbine-scale problem, as the magnitude of the turbine thrust coefficient, $C_{T L}$, is necessarily greater than the array thrust coefficient, $C_{T A}=\sigma_{1}^{2} B_{L} C_{T L}$. Consequently, the acceleration of the turbine bypass flow is greater than that in the array bypass flow, and complex non-physical solutions to the model arise in the turbine-scale model. The physical interpretation of these results is not clear, however it may be associated with supercritical flow developing in the bypass. Hence, particularly at large global blockages, there is a limited subspace of feasible solutions.

Figure 4 shows the effect of the Froude number on the thrust and power coefficients and the basin efficiency. Increasing the Froude number increases the maximum power and thrust coefficients; in the case of $B_{A}=$ $0.2, B_{L}=0.2$ (e.g., 1D lateral inter-turbine spacing in a depth of $2 \mathrm{D}$ ), $C_{P A}=0.86$ when $F r_{A}=0.05$ rises to $C_{P A}=0.89$ for $F r_{A}=0.20$. The corresponding efficiency at peak power is $\eta=0.536$ at $F r_{A}=0.05$ and increases to $\eta=0.552$ when $F r_{A}=0.20$.

The Froude number always appears as $F r_{A}^{2}$ in the model equations, and may be interpreted as the ratio of dynamic to static head in the flow. The operation of a turbine causes the relative balance of dynamic and static head to change, the extent of which is determined by the upstream Froude number and its effect on the operation of the turbine. At the turbine plane, there is a step change in static head, while the flow speed is continuous across the turbine. The wake expansion region between stations three and four is characterised by the exchange of dynamic to static head, resulting in a recovery of the static head at the expense of reducing the flow speed. The volume constraint introduced by the free surface causes an acceleration of the flow in the bypass region, which reduces the static head in the bypass. Station four is defined as the point at which the falling static head in the bypass equalises with the rising static head in the wake region after which the two flows mix between stations four and five, resulting in a uniform outflow velocity at five. The change in free surface height at the outlet relative to the inlet is due to the energy extraction by the turbine and the resulting transfer of static energy to dynamic energy as the flow accelerates to conserve mass flux in the channel. 
The rate of change of free surface height relative to total head removal is related to the Froude number by $\left(1-F_{A}^{2}\right)^{-1}$. Hence, as $\mathrm{Fr}_{A}$ is increased, the downstream height reduces more quickly for fixed total head removal, and thus the turbine bypasses are further volume flux constrained leading to greater static head drop at station four. For a given head drop, and therefore thrust, higher power is achieved by the turbine because a higher flow speed is maintained through the turbine as less acceleration is required of the flow in the bypass as Froude number increases. Correspondingly, a given level of power is achieved with a lower thrust as Froude number increases and thus lower overall power removed from the flow. Therefore efficiency increases with Froude number for a given $C_{P A}$.

\section{Limiting cases}

\subsection{Rigid lid}

The analysis presented by Nishino and Willden was for a flow with a rigid lid [7], the results of which are recovered in the limit of the present model when the Froude number tends to zero. It is assumed in the rigid lid case that the change in water depth in the channel is negligible compared to the water depth, i.e. $\delta \theta \approx 0$. Neglecting high order terms in $\delta \theta$ and rearranging terms in (15) for $\delta \theta$ yields:

$$
\delta \theta=\frac{F r_{L}^{2} B_{L} C_{T L}}{2+F r_{L}^{2}\left(B_{L} C_{T L}-2\right)},
$$

so that in taking $\mathrm{Fr}_{L}^{2} \rightarrow 0, \delta \theta \rightarrow 0$ and the rigid lid case is recovered.

As discussed in the previous section, the Froude number always appears as $F r_{L}^{2}$ and represents the relative importance of dynamic to static head in the flow. When $F r_{L}^{2} \rightarrow 0$, the dynamic energy is negligible relative to the static energy, and therefore the effect of the turbine thrust on the dynamic energy is negligible relative to the effect on the static energy. Thus, in the limit $F r_{L} \rightarrow 0$, only the hydrostatic pressure changes far downstream of the turbine, and there is no change in the flow velocity at the channel outlet, as is assumed in rigid models [7], [6].

\subsection{Infinitely wide channel}

Tidal arrays will typically have low global blockage ratios due to bathymetric variations, the need to allow for shipping and other marine activities, and practical and technical constraints on the number of turbines that can be installed. Furthermore, tidal arrays built off the coast, such as at headland sites, can be approximated as being deployed in semi-infinitely wide channels. Figure 3 indicated that at low global blockage ratios there is nevertheless a significant increase in the maximum power coefficient as the array blockage is reduced, and it is therefore of practical interest to explore the limiting case of an infinitely wide channel $\left(B_{A} \rightarrow 0\right)$.

In the case of channel width $W_{C} \rightarrow \infty$, the array occupies a relative region of the flow that becomes infinitesimally small, so that only the flow close to the array is affected by turbine operation. When $W_{C} \rightarrow \infty$, so that $B_{A}, B_{G} \rightarrow 0$, however the local blockage $B_{L}$ remains finite. It can be shown from the conservation of mass equations that $\tau_{6}, \zeta_{6}=1$, therefore the operation of the turbine array does not affect the far field flow. Considering $O(L)$ terms in the dimensional form of the momentum equation (23) between stations 0 and 6 yields, noting $\tau_{6}=1$ :

$$
T_{A}=\rho L h_{0} u_{0}^{2} \zeta_{1} \sigma_{1}\left(1-\sigma_{6}\right) ; \quad C_{T G}=2 \sigma_{1}\left(1-\sigma_{6}\right) .
$$

These equations, which complete the array scale model, are solved in conjunction with equation (15) for the inner flow to determine the overall solution.

Figure 5 shows the effect of varying the Froude number on the maximum power coefficient, with the results of the rigid lid case [7] included for comparison. Increasing the Froude number causes the maximum power coefficient to increase from the rigid lid case, for which $C_{P A, \max }=0.798$ at $B_{L} \approx 0.4$, by over $10 \%$ to $C_{P A, \max }=0.888$ at $B_{L} \approx 0.45$ when the Froude number increases to $F r_{A}=0.30$. The resulting maximum power coefficient is almost $50 \%$ above the Lanchester-Betz limit, $C_{P A, \max }=0.593$. It should be noted that, 


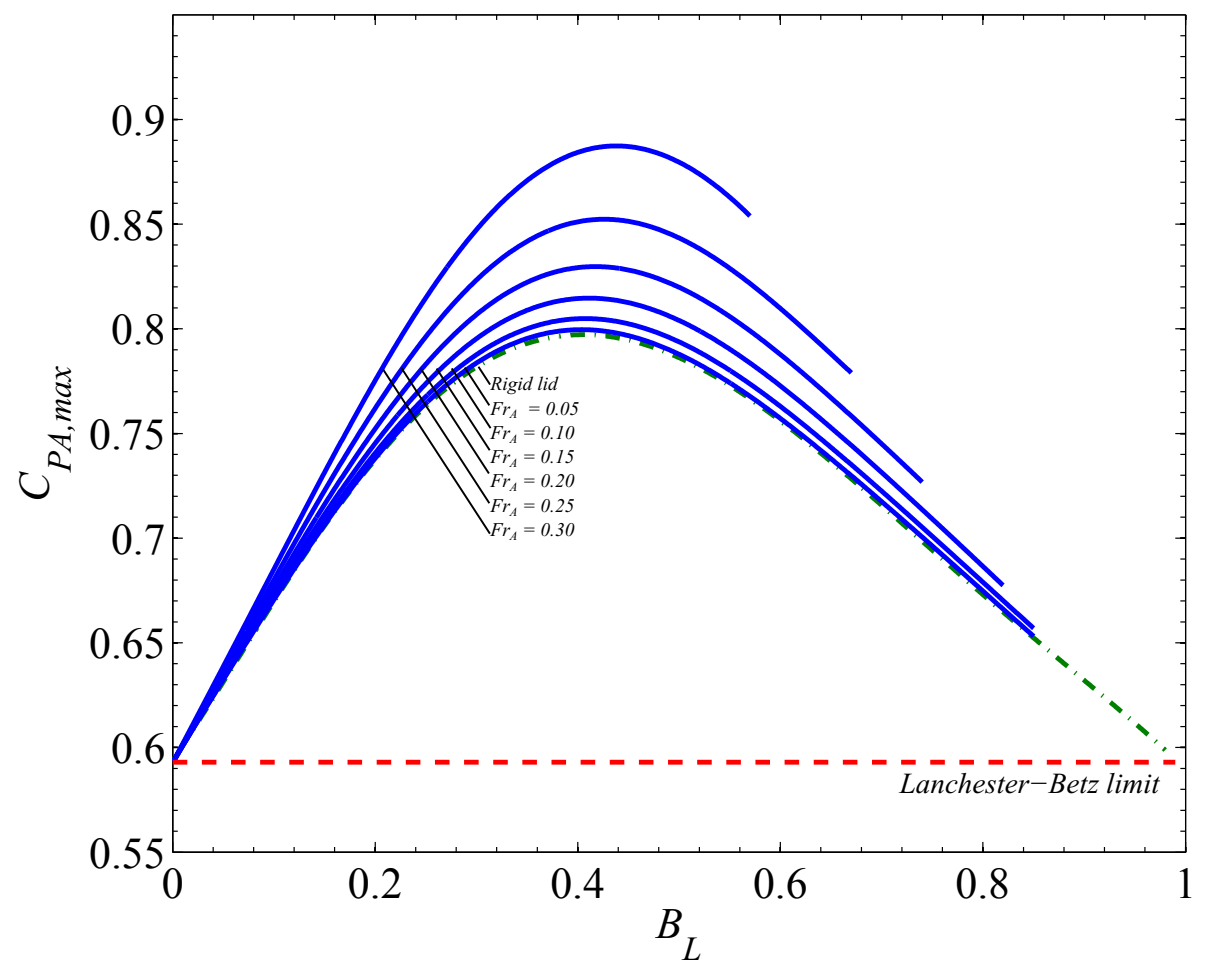

Figure 5: Effect of local blockage $B_{L}$ and Froude number $F r_{A}$ on the maximum power coefficient $C_{P A}, m a x$, compared to the rigid lid case of Nishino and Willden [7] and the Lanchester-Betz limit, $C_{P A, \max }=0.593$.

even in the rigid lid case, there is an increase in the maximum power coefficient of almost $35 \%$ above the Lanchester-Betz limit, as noted numerically by Nishino and Willden [7]. Although the channel is infinitely wide, the blockage of the turbines in the finite depth causes the Lanchester-Betz limit, derived for an infinite flow field in all directions, to be surpassed. The maxima in the $C_{P A, \max }$ curves indicate that, even when the array blockage is negligible, maximum power extraction occurs only when reasonably substantial local blockage ratios are achieved. For maximum power, $B_{L} \approx 0.4$ which corresponds for instance to an axial flow turbine of diameter $D$ operating in water of depth $1.6 D$ with $0.25 D$ intra-turbine spacing, i.e. a turbine flow passage of area $2 D^{2}$. Alternatively, it could be achieved with a horizontal axis cross flow turbine of diameter $D$ in a water depth of $2.5 D$.

\section{Conclusions}

A theoretical model has been proposed to examine the energy extraction of a finite width array of tidal turbines in a flow with a deformable free surface. It was assumed that the flow around each turbine and the flow around the array occurred on different spatial and temporal scales. The flow mixing downstream of the turbine was assumed to occur much more quickly than flow mixing downstream of the array due to the relative scale of the dimensions of the turbine diameter and array width. The two scales were coupled wherein the flow through the array provided the upstream flow speed and hydrostatic pressure boundary conditions to the turbine-scale model. The aggregate thrust of the turbines and resulting change in flow speed and hydrostatic pressure at the downstream boundary of the turbine-scale problem provided the internal boundary condition that led to the array-scale mixing problem. The two models were investigated as open channel flow problems, to which the principles of conservation of mass, momentum, and energy were applied following [11] and [3]. 
The model explored the physics of the energy extraction of an array of turbines, in particular the effect that the local, array, and global blockage has on power extraction, as well as the effect of the Froude number and thrust coefficient. The power extracted by an array of tidal turbines increases as the local blockage increases as a result of increased resistance to flow diverting around each turbine due to the presence of neighbouring turbines. As tidal current turbines cause a change in the balance of the dynamic and static head in the flow, it was also expected that the Froude number, which may be expressed as the ratio of these two quantities, should also be important. Extracted power also increases with Froude number as the deformation of the free surface increases with increasing Froude number, constraining the volume-flux in the turbine bypass and thus maintaining a higher flow speed through the turbines, resulting in a greater extracted power.

Two limiting cases of the model presented herein were also investigated; the limit of an infinitely wide channel, and the rigid-lid model. In the limit of an infinitely wide channel, it was shown that there exists an optimal blockage ratio of approximately $B_{L}=0.4$ to maximise energy extraction and that the rigid lid limit of $79.8 \%$ kinetic energy extraction of [7] further increases with increasing Froude number. At Froude numbers representative of the Pentland Firth and Cook Strait, up to $83.5 \%$ of the kinetic energy in the flow may be extracted when the array blockage is zero (when the array occupies a vanishingly small fraction of the channel cross-section), representing a $41 \%$ increase above the Lanchester-Betz limit. In reality, the non-zero, albeit small array blockage that would be achieved by a modest turbine array in these channels would slightly increase this limit. In channels of finite width, it is generally possible, for fixed global blockage and thus number of turbines, to increase the extracted power by increasing the turbine blockage, although there are geometrical constraints which limit this. It was also shown that rigid lid models are recovered in the limit $F r_{A}^{2} \rightarrow 0$ when the change in the downstream height of the flow becomes negligible and the operation of the turbine only changes the static head drop across the flow.

The leading order effect of bulk momentum reduction in the flow due to finite-length tidal stream fences has been supported through numerical simulations [8] and recent experimental work on arrays of actuator discs [2]. Although not the focus of the current work, the number of rows of turbines, and the arrangement of turbines within those rows, has been investigated by [4] and [10] with similar analytic momentum balance analyses, where [10] suggest, for example, that over 1000 turbines would be required in the Pentland Firth to harness a significant fraction of the available tidal resource. The present analysis offers tidal turbine developers a benchmark for assessing the interactions between the closely packed turbines that will be required in such developments, and in particular highlights the importance of designing turbines capable of achieving the levels of thrust required to achieve the peak power predicted by theory. As discussed in [8], the scale separation assumption is significantly influenced by the level of ambient turbulence, and further numerical and experimental analyses would be required to evaluate the practical potential of tidal turbine arrays.

\section{Acknowledgement}

The authors would like to acknowledge the support of the Oxford Martin School, University of Oxford.

[1] Brent, R. P. [1973], Algorithms for minimization without derivatives, Englewood Cliffs, NJ: Prentice Hall.

[2] Cooke, S. C., Willden, R. H. J., Byrne, B., Stallard, T. and Olczak, A. [2015], Experimental investigation of tidal turbine partial array theory using porous discs, in 'Eleventh European Wave and Tidal Energy Conference', Nantes, France.

[3] Draper, S., Houlsby, G. T., Oldfield, M. L. G. and Borthwick, A. G. L. [2010], 'Modelling tidal energy extraction in a depth-averaged coastal domain', IET Renewable Power Generation 4, 545-554.

[4] Draper, S. and Nishino, T. [2014], 'Centred and staggered arrangements of tidal turbines', Journal of Fluid Mechanics 739, 72-93.

[5] Garrett, C. and Cummins, P. [2005], 'The power potential of tidal currents in channels', Proceedings of the Royal Society: A 461, 2563-2572. 
[6] Garrett, C. and Cummins, P. [2007], 'The efficiency of a turbine in a tidal channel', Journal of Fluid Mechanics 588, 243-251.

[7] Nishino, T. and Willden, R. H. J. [2012], 'The efficiency of an array of tidal turbines partially blocking a wide channel', Journal of Fluid Mechanics 708, 596-606.

[8] Nishino, T. and Willden, R. H. J. [2013], 'Two-scale dynamics of flow past a partial cross-stream array of tidal turbines', Journal of Fluid Mechanics 730, 220-244.

[9] Vennell, R. [2010], 'Tuning turbines in a tidal channel', Journal of Fluid Mechanics 663, 253-267.

[10] Vennell, R., Funke, S. W., Draper, S., Stevens, C. and Divett, T. [2015], 'Designing large arrays of tidal turbines: A synthesis and review', Renewable and Sustainable Energy Reviews 41, 454-472.

[11] Whelan, J. I., Graham, J. M. R. and Pieró, J. [2009], 'A free surface and blockage correction for tidal turbines', Journal of Fluid Mechanics 624, 281-291. 